\title{
Utility of a Computed Tomography-Based Navigation System (O-Arm) for En Bloc Partial Vertebrectomy for Lung Cancer Adjacent to the Thoracic Spine: Technical Case Report
}

\author{
Kazuyoshi Kobayashi ${ }^{1}$, Shiro Imagama ${ }^{1}$, Zenya $\operatorname{Ito}^{1}$, Kei Ando ${ }^{1}$, Kohei Yokoi ${ }^{2}$, Naoki Ishiguro ${ }^{1}$ \\ ${ }^{1}$ Department of Orthopedic Surgery, Nagoya University Graduate School of Medicine, Nagoya, Japan \\ ${ }^{2}$ Department of Thoracic Surgery, Nagoya University Graduate School of Medicine, Nagoya, Japan
}

We describe successful vertebrectomy from a posterior approach using a computed tomography (CT)-based navigation system (0-arm) in a 53-year-old man with adenocarcinoma of the posterior apex of the right lung with invasion of the adjacent rib, thoracic wall, and T2 and T3 vertebral bodies. En bloc partial vertebrectomy for lung cancer adjacent to the thoracic spine was planned using 0-arm. First, laminectomy was performed from right $\mathrm{T} 2$ to $\mathrm{T} 3$, and pedicles and transverse processes of $\mathrm{T} 2$ to $\mathrm{T} 3$ were resected. 0 -arm was used to confirm the location of the cutting edge in the T2 to 3 right vertebral internal body, and osteotomy to the anterior cortex was performed with a chisel. Next, the patient was placed in a left decubitus position. The surgical specimen was extracted en bloc. This case shows that 0 -arm can be used reliably and easily in vertebrectomy from a posterior approach and can facilitate en bloc resection.

Keywords: Lung neoplasms; Computed tomography-based navigation system; En bloc partial vertebrectomy

\section{Introduction}

Treatment of non-small-cell lung cancer (NSCLC) with vertebral body invasion is challenging and is the current goal of several innovative surgical techniques. NSCLC with substantial invasion of vertebral bodies used to be a contraindication for surgery due to poor long-term survival [1]. However, en bloc resection of NSCLC after induction chemoradiotherapy has long-term survival with acceptable morbidity and a 5-year predicted survival rate of $43 \%-74 \%$ [2-6].

$\mathrm{O}$-arm (Medtronic Inc., Minneapolis, MN, USA) is an intraoperative full-rotation, multidimensional imaging system and a new computer-assisted surgery device. Oarm with a navigation system generates real-time multiimages and has an advantage for placement of pedicle screws [7-9]. Here, we report our successful use of O-arm in en bloc partial vertebrectomy for lung cancer. There have been no previous reports of such a procedure using $\mathrm{O}$-arm. Use of this device permitted accurate confirmation of the surgical margins of osteotomy, and it was possible to perform osteotomy clearly and easily from a posterior approach.

The study was approved by the ethics committee of Nagoya University Graduate School of Medicine. The patient gave informed consent to the collection and use of data

\footnotetext{
Received Sep 13, 2015; Revised Oct 8, 2015; Accepted Oct 8, 2015

Corresponding author: Shiro Imagama

Department of Orthopedic Surgery, Nagoya University Graduate School of Medicine,

65 Tsurumai Showa-ku, Nagoya, Aichi 466-8550, Japan

Tel: +81-52-833-6021, Fax: +81-52-833-6021, E-mail: imagama@med.nagoya-u.ac.jp
} 
for research purposes.

\section{Case Report}

A 61-year-old man was found to have adenocarcinoma of the posterior apex of the right lung with invasion of the adjacent rib, thoracic wall, and T2 and T3 vertebral bodies, as shown on chest CT and magnetic resonance imaging (MRI) (Fig. 1). No distant metastasis was evident on abdominal CT, brain MRI, bone scan, and FDG-positron emission tomography, and thus the pretreatment TNM staging was IIIA (T4N0M0). Chemotherapy with two cycles of cisplatin and vinorelbine was conducted, together with radiotherapy at a dose of $40 \mathrm{~Gy}$ (2 Gy/day, 5 day/ week). On subsequent chest CT, the tumor had shrunk by $25 \%$. Restaging confirmed persistent T4NOM0 and en bloc resection was planned two months after performance of chemoradiotherapy.

The surgical procedure was performed in two steps. First, the patient was placed in a prone position on a
Jackson radiolucent table with a Mayfield head clamp, and a median posterior incision was made from $\mathrm{C} 7$ to $\mathrm{T} 4$. Pedicle screws were inserted at left $\mathrm{T} 1$ to $\mathrm{T} 4$, and right $\mathrm{T} 1$ and $\mathrm{T} 4$, and laminectomy from the right $\mathrm{T} 2$ to $\mathrm{T} 3$ was performed, followed by left rod fixation. On the right side, the pedicles and transverse processes from $\mathrm{T} 2$ to $\mathrm{T} 3$ were resected (Fig. 2A), the costovertebral joints were disarticulated, and the right $\mathrm{T} 2$ and $\mathrm{T} 3$ roots were dissected. After a reference frame was clamped to the exposed spinous process, an intraoperative CT scan was obtained sterilely with $\mathrm{O}$-arm. With reference to the intraoperative CT data, the navigation system then generated real-time axial, coronal, and sagittal images that allowed confirmation of the location of the cutting edge in the T2-3 right vertebral internal bodies. Using the margin from the tumor defined in the preoperative plan, osteotomy was performed with a chisel to the anterior cortex (Figs. 2B, 3) and then right rod fixation was performed.

Second, the patient was placed in a left decubitus position, and a long posterior thoracotomy incision was made

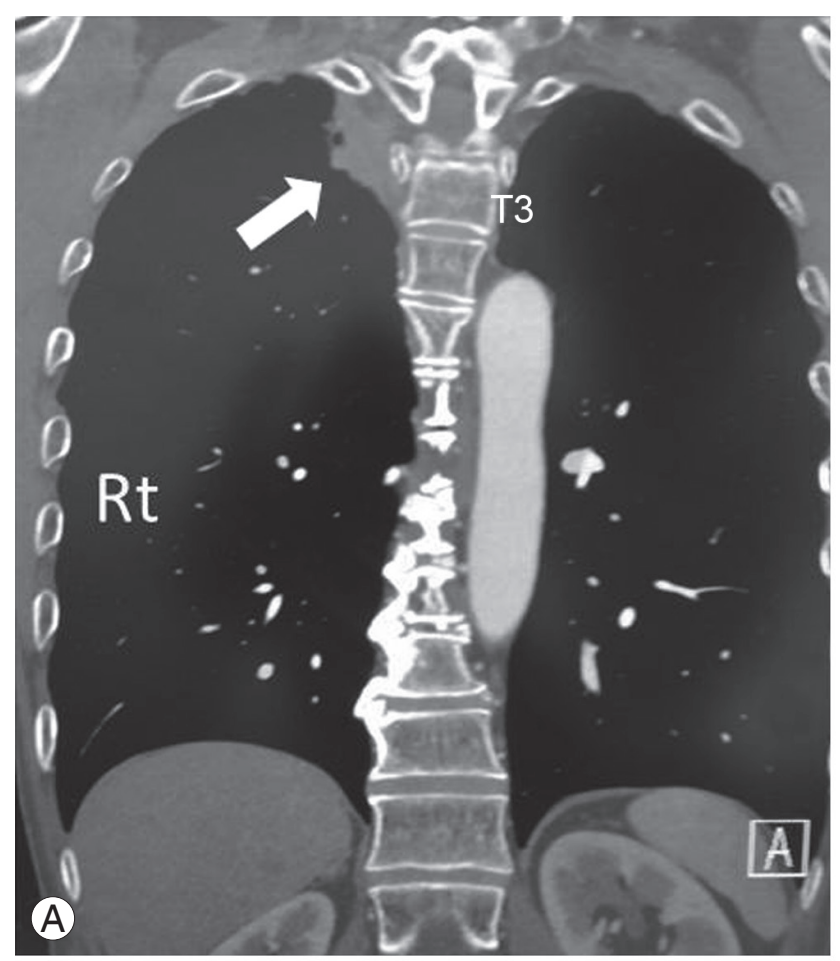

Fig. 1. Computed tomography showing a tumor in the right upper lobe adjacent to T2 and T3 in coronal (A) and axial (B) views. Magnetic resonance imaging showing a tumor in the right upper lobe adjacent to vertebra in an axial view (C).
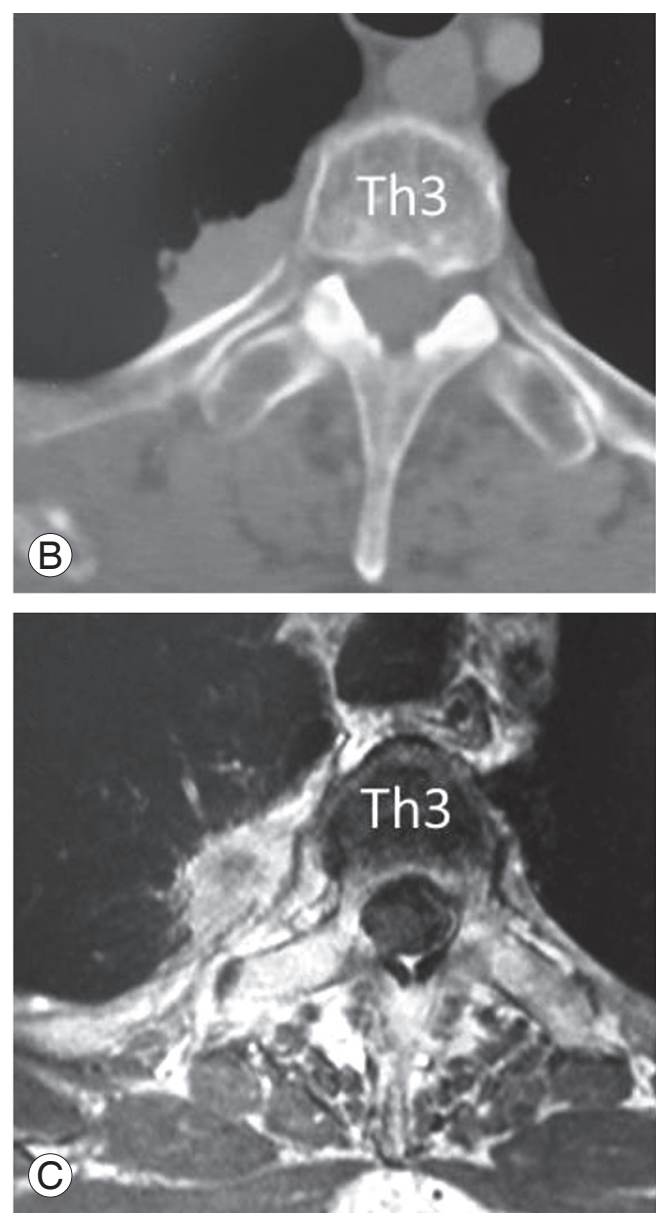


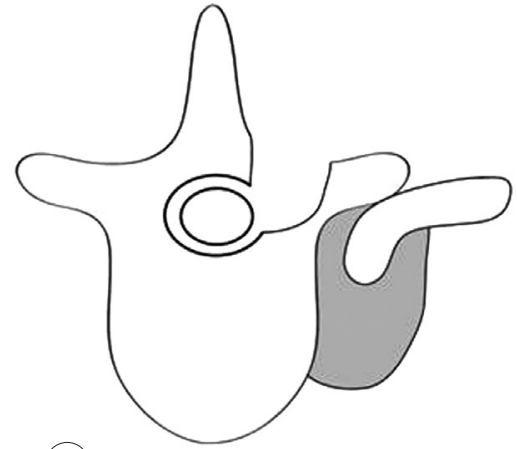

(A)

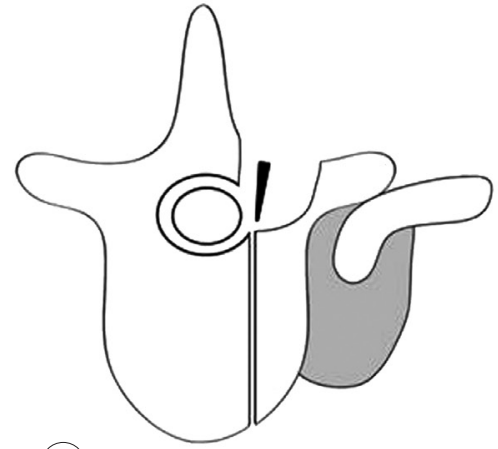

(B)

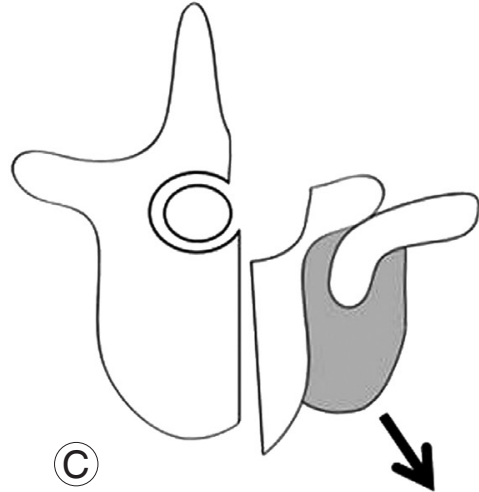

C)

Fig. 2. (A) Laminectomy of the right side was performed and pedicles and transverse processes were resected. (B) Osteotomy was performed with a chisel using a posterior approach. (C) The attached tumor, lung and right chest wall were extracted forward en bloc using an anterior approach.
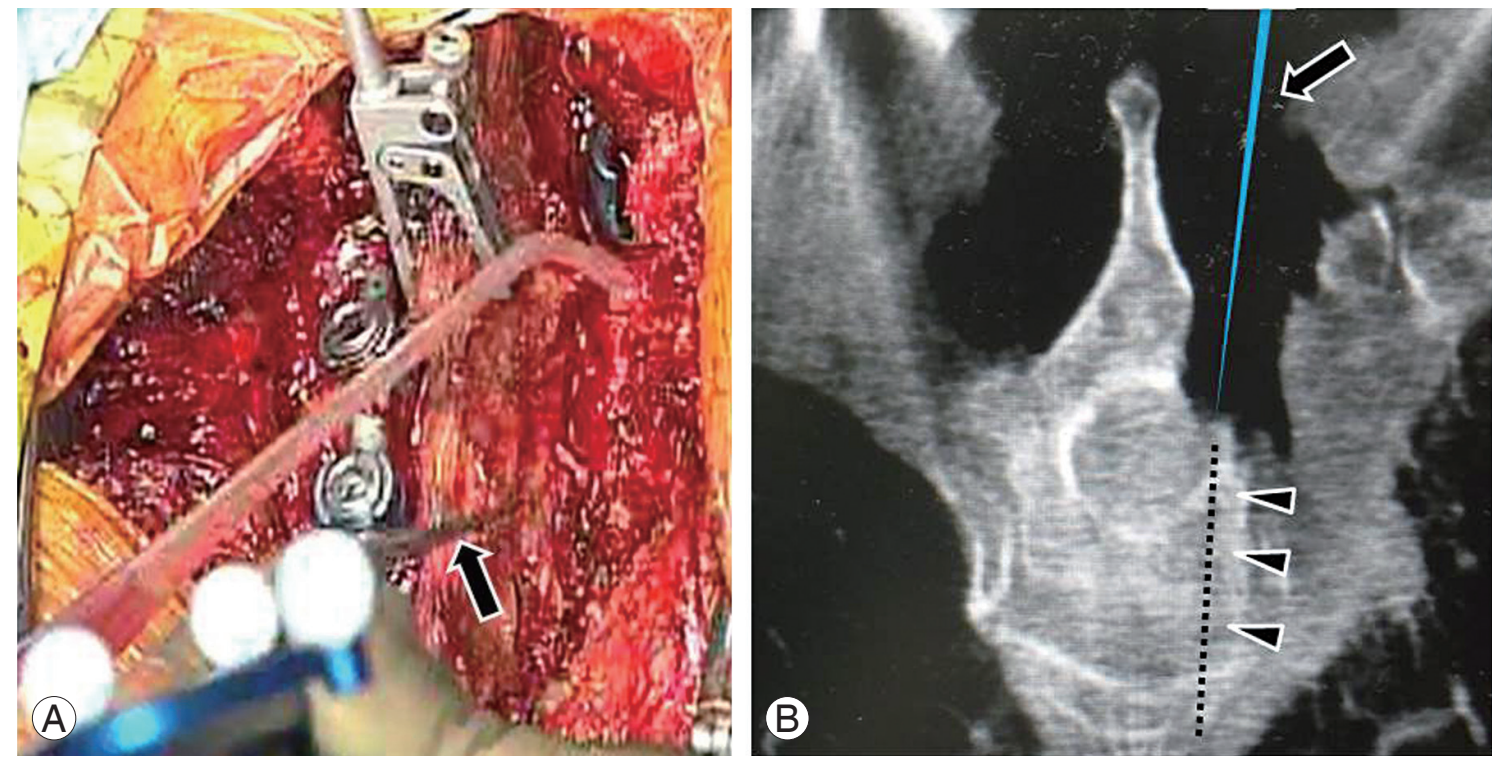

Fig. 3. (A) Intraoperative photograph to confirm the osteotomy line with navigation using a probe (arrowhead, navigation probe). (B) Virtual osteotomy line and probe in computed tomography imaging (triangle, osteotomy line; arrowhead, navigation probe).

as a gentle arc from the spinous process of $\mathrm{T} 2$ to the inferior angle of the scapula. The right second and third ribs were resected in thoracotomy, with right upper lobectomy of the lung (Fig. 2C). A surgical specimen including vertebral bodies (T2 and T3), attached tumor, right upper lobe, and chest wall was extracted en bloc (Fig. 4). Finally, we confirmed hemostasis and closed the thoracotomy. The operation time was 493 minutes and the estimated blood loss was $1,242 \mathrm{~mL}$.

Pathologic examination confirmed complete resection of adenocarcinoma adjacent to the $\mathrm{T} 2$ and $\mathrm{T} 3 \mathrm{ribs}$ and vertebral body, which were not infiltrated. There have been no problems in the postoperative course and the patient has shown no findings indicating recurrence (Fig. 5).

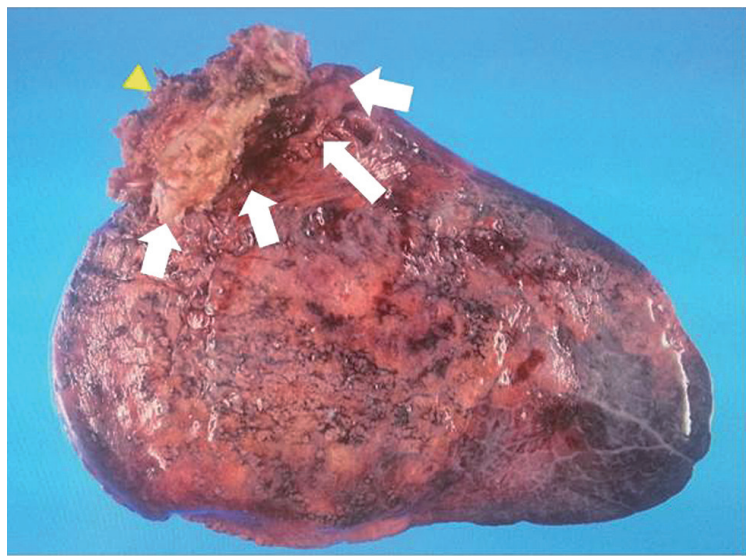

Fig. 4. En bloc resected specimen including the body of the $T 2$ and $\mathrm{T} 3$ vertebra, attached tumor and right upper lobe. Triangle, resected vertebra; arrow head, tumor. 

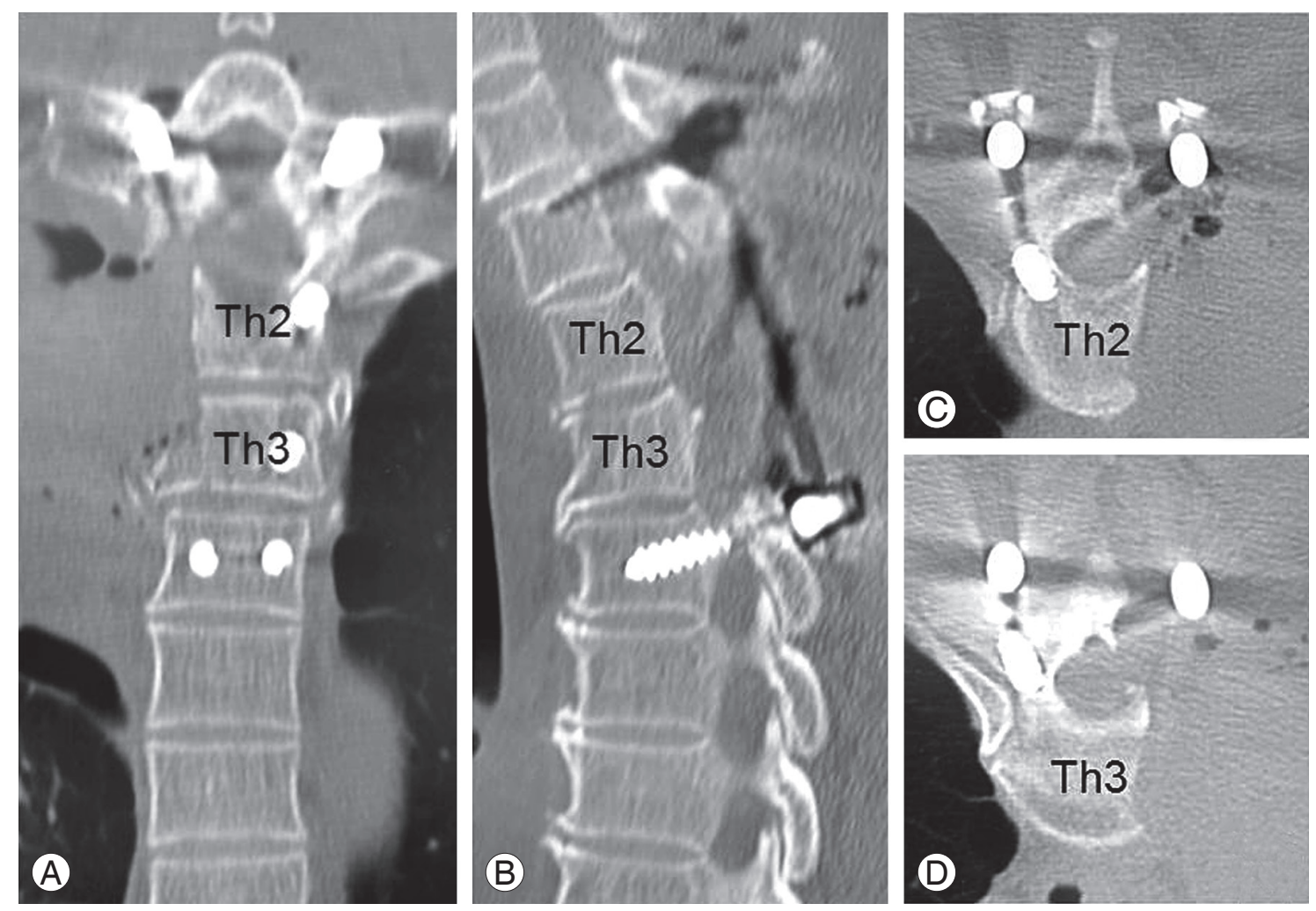

Fig. 5. Postoperative computed tomography after en bloc partial vertebrectomy. (A) Coronal view. (B) Sagittal view. (C) Axial view at T2. (D) Axial view at T3.

\section{Discussion}

Introduction of spinal surgery techniques into lung cancer operations has improved the prognosis of lung cancer with vertebral invasion $[2,10,11]$. En bloc resection of NSCLC can be achieved with acceptable morbidity and a 5-year predicted survival rate of $43 \%-74 \%$ [2-6].

Yokomise et al. [6] described three procedures for vertebrectomy for lung cancer invading the spine: transverse resection (oblique osteotomy from posterior to the thoracic cavity), one-third resection (osteotomy from posterior to the canal, and subsequently from the thoracic cavity to the canal), and half resection (after laminectomy, osteotomy from the thoracic cavity to the canal). Each procedure is selected depending on the tumor size and extent of invasion, with reconstruction of the body recommended in more than half of vertebrectomies $[10,12]$.

Total en bloc spondylectomy (TES) is a useful procedure for metastasis to the spine [13]. TES is suitable in a case in which resection of more than half of the vertebral body is required, and bleeding is less than that in partial vertebrectomy. However, in the current case, less than half of the vertebral body was resected, vertebral body reconstruction was not required, and radiotherapy was performed. Therefore, en bloc partial vertebrectomy was considered desirable based on the reduced risk of nonunion and rod breakage.

$\mathrm{O}$-arm has utility for screw insertion in spine surgery $[7,9,14,15]$. Santos et al. [14] showed that intraoperative $\mathrm{O}$-arm images can accurately detect significant pedicle screw violations in the thoracic and lumbosacral spine. Shimizu et al. [15] reported that O-arm is useful for accurate pedicle screw placement in patients with scoliosis, and Ishikawa et al. [7] also evaluated the reliability of cervical pedicle screw placement using $\mathrm{O}$-arm. O-arm has also been used in surgery other than spine surgery, such as in closed reduction of posterior sternoclavicular dislocations, arthroscopic glenohumeral arthrodesis, and videoassisted thoracoscopic surgery [16-18]. However, there are no previous reports of use of O-arm in osteotomy for en bloc vertebrectomy with a posterior approach.

Grunenwald et al. [10] found that en bloc resection of lung cancer invading a vertebral body is complex and difficult to perform. Without use of O-arm, osteotomy of 
the anterior column might have to be performed via an anterior approach for en bloc partial vertebrectomy for a paravertebral tumor, after laminae and pedicle resection and screw fixation in a posterior approach. In such a procedure, it is difficult to confirm surgical margins for resection, and there would have been increased invasiveness due to the difficulty of en bloc partial vertebrectomy. In contrast, with intraoperative navigation using $\mathrm{O}$-arm, as shown in the current case, it is easy to confirm the cutting edge with a navigation probe. A further advantage is that the intraoperative depth during osteotomy can be confirmed accurately and instantly using O-arm. Vertebrectomy can then be performed reliably with a chisel from a posterior approach. This procedure makes anterior osteotomy unnecessary and simplifies en bloc vertebrectomy. In addition, using $\mathrm{O}$-arm, we were able to confirm the placement of pedicle screws intraoperatively.

In conclusion, we performed en bloc partial vertebrectomy for lung cancer adjacent to a thoracic vertebral body using the $\mathrm{O}$-arm system. This case shows that $\mathrm{O}$-arm can be used reliably and easily in vertebrectomy from a posterior approach to facilitate en bloc resection.

\section{Conflict of Interest}

No potential conflict of interest relevant to this article was reported.

\section{References}

1. Ginsberg RJ, Martini N, Zaman M, et al. Influence of surgical resection and brachytherapy in the management of superior sulcus tumor. Ann Thorac Surg 1994;57:1440-5.

2. DeMeester TR, Albertucci M, Dawson PJ, Montner SM. Management of tumor adherent to the vertebral column. J Thorac Cardiovasc Surg 1989;97:373-8.

3. Rao J, Sayeed RA, Tomaszek S, Fischer S, Keshavjee S, Darling GE. Prognostic factors in resected satellitenodule T4 non-small cell lung cancer. Ann Thorac Surg 2007;84:934-8.

4. Schirren J, Donges T, Melzer M, Schonmayr R, Eberlein M, Bolukbas S. En bloc resection of non-smallcell lung cancer invading the spine. Eur J Cardiothorac Surg 2011;40:647-54.

5. Yildizeli B, Dartevelle PG, Fadel E, Mussot S, Chapelier A. Results of primary surgery with T4 non-small cell lung cancer during a 25-year period in a single center: the benefit is worth the risk. Ann Thorac Surg 2008;86:1065-75.

6. Yokomise H, Gotoh M, Okamoto T, et al. En bloc partial vertebrectomy for lung cancer invading the spine after induction chemoradiotherapy. Eur J Cardiothorac Surg 2007;31:788-90.

7. Ishikawa Y, Kanemura T, Yoshida G, et al. Intraoperative, full-rotation, three-dimensional image (O-arm)based navigation system for cervical pedicle screw insertion. J Neurosurg Spine 2011;15:472-8.

8. Nottmeier EW, Young PM. Image-guided placement of occipitocervical instrumentation using a reference arc attached to the headholder. Neurosurgery 2010; 66:138-42.

9. Yoshida G, Kanemura T, Ishikawa Y. Percutaneous pedicle screw fixation of a hangman's fracture using intraoperative, full rotation, three-dimensional image (o-arm)-based navigation: a technical case report. Asian Spine J 2012;6:194-8.

10. Grunenwald DH, Mazel C, Girard P, et al. Radical en bloc resection for lung cancer invading the spine. J Thorac Cardiovasc Surg 2002;123:271-9.

11. Sause W, Kolesar P, Taylor SI, et al. Final results of phase III trial in regionally advanced unresectable non-small cell lung cancer: Radiation Therapy Oncology Group, Eastern Cooperative Oncology Group, and Southwest Oncology Group. Chest 2000;117:35864.

12. Gandhi S, Walsh GL, Komaki R, et al. A multidisciplinary surgical approach to superior sulcus tumors with vertebral invasion. Ann Thorac Surg 1999; 68:1778-84.

13. Murakami H, Kawahara N, Demura S, Kato S, Yoshioka K, Tomita K. Total en bloc spondylectomy for lung cancer metastasis to the spine. J Neurosurg Spine 2010;13:414-7.

14. Santos ER, Ledonio CG, Castro CA, Truong WH, Sembrano JN. The accuracy of intraoperative O-arm images for the assessment of pedicle screw postion. Spine (Phila Pa 1976) 2012;37:E119-25.

15. Shimizu M, Takahashi J, Ikegami S, Kuraishi S, Futatsugi T, Kato H. Are pedicle screw perforation rates influenced by registered or unregistered vertebrae in multilevel registration using a CT-based navigation system in the setting of scoliosis? Eur Spine J 2014;23: 2211-7. 
16. Ladermann A, Denard PJ. Arthroscopic glenohumeral arthrodesis with o-arm navigation. Arthrosc Tech 2014;3:e205-9.

17. Ohtaka K, Takahashi Y, Kaga K, Senmaru N, Kotani Y, Matsui Y. Video-assisted thoracoscopic surgery using mobile computed tomography: new method for locating of small lung nodules. J Cardiothorac Surg 2014;9:110.

18. Sullivan JP, Warme BA, Wolf BR. Use of an O-arm intraoperative computed tomography scanner for closed reduction of posterior sternoclavicular dislocations. J Shoulder Elbow Surg 2012;21:e17-20. 